\title{
DIE WILHELM WEITLING PAPERS
}

\author{
NEUE QUELLEN UND DOKUMENTE AUS DEM NACHLASS \\ WILHELM WEITLINGS IN DER PUBLIC LIBRARY, NEW \\ YORK
}

Anzuzeigen ist ein umfangreicher Quellenbestand der Manuscript Division der New York Public Library über Wilhelm Weitling (1808-71). Dessen Nachlaß galt in der deutschsprachigen Literatur bisher als verschollen; Hinweisen auf die Existenz dieses Nachlasses, die in der Biographie von Carl Wittke seit 1950 vorlagen, ist die Forschung bisher nicht nachgegangen. ${ }^{1}$ Die Bedeutung dieser neuen Dokumente für die Sozialismusforschung, die Historiographie zur Geschichte der Arbeiterbewegung, für die politische Theorie und Wissenschaftsgeschichte ergibt sich aus Weitlings historischer Stellung als Arbeiterkommunist und erster Theoretiker und Organisator der deutschen Arbeiterbewegung. Als Zeitgenosse von Karl Marx markiert Weitling einen Schnittpunkt in den Entwicklungslinien von ,utopischem” und ,wissenschaftlichem” Sozialismus.

Weitlings Wirken in der europäischen Arbeiterbewegung vor 1848 ist weitgehend bekannt, selten beschäftigt sich die Literatur allerdings mit seiner Tätigkeit in Amerika, wo Weitling Ende des Jahres 1846 erstmals eintraf. Vorausgegangen war sein Zerwürfnis mit dem Bund der Gerechten in London und mit dem von Marx und Engels geleiteten Kommunistischen Korrespondenzkomitee in Brüssel. Weitling hatte sich geweigert, das Zirkular gegen Hermann Kriege zu unterzeichnen, woraufhin ihm dieser die Stelle eines Redakteurs beim Volks-Tribun in New York anbot. Als Weitling in New York eintraf, hatte die Zeitung jedoch bereits Konkurs angemeldet.

Bei Ausbruch der europäischen Revolutionen kam er als Delegierter des in Amerika gegründeten Befreiungsbundes sofort nach Europa zurück, ohne in Berlin, Köln, Hamburg und Altona große Wirkung entfalten zu

1 Carl Wittke, The Utopian Communist. A Biography of Wilhelm Weitling, Nineteenth-Century Reformer, Baton Rouge 1950. 
können. ${ }^{2}$ Als die Gegenrevolution triumphierte und die Existenz des von Weitling in Hamburg und Altona verbreiteten Befreiungsbundes bekannt wurde, entzog er sich dem Zugriff der Behörden im August 1849 durch die diesmal endgültige Emigration nach Amerika. Dieses Exil war zwar nicht selbstgewählt, mündete deswegen aber nicht in Resignation und war keineswegs mit politischer und theoretischer Abstinenz verbunden. Weitling hat von 1849 bis zu seinem Tode meist in New York gelebt und im Rahmen seiner Möglichkeiten unter immer schlechter werdenden materiellen Bedingungen nie aufgehört, politisch-praktisch und theoretisch für seine ungebrochene Überzeugung von der Notwendigkeit einer besseren und sozialistischen Welt einzutreten.

Ab Januar 1850 gab er die Republik der Arbeiter heraus, die zeitweise wöchentlich erschien und eine maximale Auflagenstärke von mehr als 4000 Exemplaren erreichte. Vom 22. bis 28. Oktober tagte in Philadelphia der erste deutsch-amerikanische Arbeiterkongre $B$, zu dem insgesamt ca 4400 nach Gewerben organisierte Arbeiter u.a. aus Baltimore, Buffalo, Cincinnati, Louisville, Newark, New York, Philadelphia, Pittsburg und St Louis ihre Delegierten entsandt hatten. Im gleichen Jahr nahm Weitling Kontakt auf mit der Kolonie Communia in Clayton County, Iowa. Er gliederte die Kolonie dem von ihm gegründeten Arbeiterbund an und ließ sich infolge zunehmender Schwierigkeiten der Kolonie im November 1853 $\mathrm{zu}$ deren Verwalter wählen. Indes war der Niedergang aller politischen Unternehmungen Weitlings nicht mehr aufzuhalten. Bereits im Januar 1854 legte er die Verwalterstelle im Streit mit den Kommunarden nieder, im April 1855 erschien die letzte Ausgabe der Republik der Arbeiter; damit war auch der Arbeiterbund gescheitert.

1854 heiratete Weitling die Deutsche Caroline Toedt. Er lebte mit seiner Frau, den schließlich sechs Kindern und einer Schwägerin im deutschen Viertel in New York. ${ }^{3}$ Die ganze Familie arbeitete hausgewerblich in der Schneiderei. Eine zeitweilige Tätigkeit bei der Einwanderungsbehörde in Castle Garden mußte Weitling am 11. Juni 1862 aufgeben, weil er sich geweigert hatte, unter den Einwanderern Rekruten für die Bürger-

\footnotetext{
2 Weitlings Rolle in der Revolution von 1848-49 ist bislang in der Literatur nur unzureichend dargestellt. Über seine Tätigkeit in Hamburg und Altona vgl. Hans-Arthur Marsiske, „Theorierezeption in der frühen deutschen Arbeiterbewegung - am Beispiel Wilhelm Weitling", Diplomarbeit, maschinenschriftlich, Hamburg 1981. Eine mit neuen Dokumenten belegte Neuinterpretation über Weitlings Rolle in der Revolution 1848-49 haben Waltraud Seidel-Höppner und Jakov Rokitjanskij vorgelegt; demnächst im Jahrbuch für Geschichte.

${ }^{3}$ Aus der Korrespondenz Weitlings lassen sich folgende Adressen in New York ermitteln: 195 Avenue B, 2919 th Street, 126 Norfolk Street, 360 Grand Street und 178 Stanton Street.
} 
kriegsarmee anzuwerben. Der Niedergang des Schneidergewerbes und die zunehmende soziale Verelendung hielten ihn nicht davon ab, an der Denkund Sprachlehre weiterzuarbeiten, die er bereits 1843-44 im Züricher Gefängnis gedanklich konzipiert hatte. Daneben beschäftigten ihn ab 1855 zunehmend astronomische Probleme. Außerdem entwickelte Weitling einige Neuerungen auf dem Gebiet der Nähmaschinentechnik. Wilhelm Weitling starb am 25. Januar 1871 in bitterer Armut, isoliert von der in Europa sich langsam zur Massenbewegung ausweitenden Arbeiterbewegung, an deren Konstituierungsphase er maßgeblich beteiligt gewesen ist. Ungebrochen stand er zu den politischen Idealen dieser Bewegung, drei Tage vor seinem Tod nahm er an einem Verbrüderungsfest der Internationale gegen den deutsch-französischen Krieg teil.

Obwohl Weitling zwischen 1849 und 1871 den überwiegenden Teil seines politisch aktiven Lebens in Amerika verbrachte, wird dieser Lebensabschnitt in der Literatur meist ausgeblendet. Dort, wo Weitlings Tätigkeit in Amerika überhaupt Erwähnung findet, geschieht dies in einer für die Weitling-Forschung seltenen Einmütigkeit durchgängig negativ. Weitlings politische Bedeutung, angefangen von seiner Rolle während der 48er Revolution, seine Bedeutung für die Konstituierung einer Arbeiterbewegung in Amerika, die Geschichte des Arbeiterbundes und der Gewerbetauschbank, bis hin zur Geschichte der Kolonie Communia, wird unterschätzt. Seine theoretischen Arbeiten aus der Zeit des amerikanischen Exils, die Denk- und Sprachlehre und die astronomischen Studien um die Mechanik des Himmels herum, galten als verschollen, Inhalt und Intention dieser Arbeiten sind verkannt worden.

Durchgängiges Merkmal der spärlichen Hinweise auf Weitling in Amerika ist seine Pathologisierung. Im ersten deutschsprachigen Beitrag nach Weitlings Tod schreibt ein anonymer Autor in der von Karl Höchberg herausgegebenen sozialistischen Revue Die Zukunft, Weitling sei ,in seinen letzten Lebensjahren geistig mehr oder weniger gestört" gewesen. ${ }^{4}$ Für den ersten Biographen Emil Kaler haben sich in den nach 1849 entstandenen theoretischen Schriften fixe Ideen ,krankhaft verkörpert”. ${ }^{5}$ Morris Hillquit, der 1906 über Weitlings Tätigkeit berichtete, schreibt: „Gegen Ende seines Lebens steigerte sich seine Überzeugung von dem

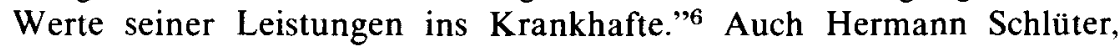

4 „Wilhelm Weitling und sein System der Harmonie und Freiheit”, in: Die Zukunft, Berlin, Jg. 1 (1877-78), S. 583-94, 606-15, Zitat S. 585.

5 Emil Kaler, Wilhelm Weitling. Seine Agitation und Lehre im geschichtlichen Zusammenhange dargestellt, Hottingen-Zürich 1887, S. 75.

${ }_{6}$ Morris Hillquit, Geschichte des Sozialismus in den Vereinigten Staaten, Stuttgart 1906, S. 155. 
dessen Arbeit 1907 erschien und der darin erstmals vorher unbekannte Quellen über Weitling in Amerika ausgewertet hat, attestierte Weitling eine „krankhafte Größe”. ${ }^{7}$ Auf der Grundlage dieser Quellen- und Literaturlage nimmt es nicht Wunder, daß auch Franz Mehring, der damals beste Weitling-Kenner in der Sozialdemokratie, in der Einleitung zur Neuherausgabe der Garantien der Harmonie und Freiheit $1908 \mathrm{zu}$ dem harten und so nicht haltbaren Urteil kam: „Er verrannte sich völlig in seine Utopistereien"; 8 die astronomischen Studien sind Mehring nichts als „leerer Wahn".

Die Pathologisierung Weitlings in der älteren Literatur ist in der jüngeren Literatur entweder kritiklos übernommen worden oder sie hat zur Ignoranz gegenüber Weitlings Tätigkeit im amerikanischen Exil geführt. Thilo Ramms Diktum, Weitlings Wirken in Amerika sei für die allgemeine Entwicklung des Sozialismus ohne Bedeutung, ist konstitutiv geworden. ${ }^{10}$ Die aus angebbaren Gründen sinnvolle historische Begrenzung des vormarxistischen kritisch-utopischen Sozialismus auf die Zeit vor 1848 darf indes nicht dazu führen, die nachfolgende Entwicklung schlichtweg nicht mehr zur Kenntnis zu nehmen. ${ }^{11}$ Die Auswertung des hier angezeigten Quellenbestandes über Weitlings politische und theoretische Arbeit in Amerika wird zeigen, daß eine Bewertung auch für die Zeit vor 1848 nur dann möglich ist, wenn die Quellen für die Zeit nach Überschreiten des Höhepunktes seiner Wirksamkeit und seines politischen und theoretischen Führungsanspruches zur Kenntnis genommen und interpretiert werden. Einige der New Yorker Materialien beziehen sich unmittelbar auf Weitlings Tätigkeit in Europa, andere lassen Bekanntes retrospektiv in neuem Licht erscheinen.

Die in der Literatur festgeschriebene Überzeugung, Weitlings Tätigkeit in Amerika sei politisch bedeutungslos und theoretisch wirr gewesen, hat

\footnotetext{
7 Hermann Schlüter, Die Anfänge der deutschen Arbeiterbewegung in Amerika, Stuttgart 1907, S. 121.

${ }^{8}$ Franz Mehring, ,Einleitung zu Wilhelm Weitling: Garantien der Harmonie und Freiheit", in Gesammelte Schriften, Berlin 1960-69, Bd 4, S. 119.

9 Ebd., S. 124.

${ }^{10}$ Thilo Ramm, Die großen Sozialisten als Rechts- und Staatsphilosophen, Stuttgart 1955, S. 484.

11 Die Revolution von 1848-49 ist als historische Zäsur für den kritisch-utopischen vormarxistischen Sozialismus allgemein anerkannt. Vgl. dazu Joachim Höppner und Waltraud Seidel-Höppner, Von Babeuf bis Blanqui, Leipzig 1975; Ahlrich Meyer, Frühsozialismus. Theorien der sozialen Bewegung 1789-1848, Freiburg, München 1977; Thilo Ramm, Der Frühsozialismus. Quellentexte, Stuttgart 1956. Zur ausführlichen Begründung dieser Zäsur vgl. Manfred Hahn, Die methodische Erforschung des vormarxistischen Sozialismus. Klärungen und Beiträge zu dessen ausstehender Quellenund Schrifttumkunde, Bremen 1982.
} 
die unzureichende Quellenlage, auf der dieses Urteil basiert, erfolgreich kaschiert und tatsächlich vorhandene Quellen unter einem festgefügten Interpretat verdeckt. Der Historiker sucht dort nicht mehr, wo kein Interesse besteht etwas zu finden, weil vermeintlich bereits alles gesagt ist. Dabei war es zunächst sicherlich nicht ganz leicht, Quellen über den in Vergessenheit geratenen Weitling aufzufinden. In dem bereits erwähnten Artikel aus der Zukunft heißt es zur Quellenlage:

Leider ist es uns nicht möglich, eingehend über die Person und das Leben Weitlings berichten zu können. Da derselbe in Deutschland fast unbekannt ist, wandten wir uns um Auskunft nach seinem letzten Aufenthaltsorte, New-York, leider ohne Erfolg. Einer der dortigen Parteigenossen hatte zwar nach dem Tode Weitlings dessen Papiere an sich gebracht, um aus denselben Mittheilungen an die Öffentlichkeit gelangen zu lassen, allein es ist dazu nicht rechtzeitig gekommen, die Papiere haben der Familic: Weitlings zurückgegeben werden müssen, und diese ist nun zur Zeit ganz verschollen. Wir theilen deshalb das Wenige mit, was uns bekannt ist. ${ }^{12}$

Knapp zehn Jahre später hat Kaler diesen Quellenstand 1887 bestätigt: „seine Witwe besaß viele Manuskripte von ihm, sie ist aber später verschollen und mit ihr der literarische Nachlaß Weitling's."13 Hermann Schlüter, der während der Zeit des Sozialistengesetzes den Buchverlag der deutschen Sozialdemokratie in Hottingen-Zürich geleitet hatte und in dieser Zeit maßgeblich am Aufbau des sozialdemokratischen Parteiarchivs beteiligt gewesen war, hat die Familie Weitlings in New York wieder aufgespürt. Schlüter hat für seine Arbeit nicht nur im Familienbesitz befindliche Dokumente einsehen dürfen, sondern auch mit Weitlings Witwe Caroline und dem ältesten Sohn Wanganschi Gespräche geführt. Schlüter hat als erster ausführlicher über Weitling in Amerika berichtet, er hat aber offenbar nicht alles Material aus dem heutigen Bestand in der Public Library eingesehen. So hat er u.a. berichtet, Weitling habe während einer Krankheit 1869 ,alle seine Manuskripte mit Ausnahme seines astronomischen Werkes und den größten Teil seiner Briefe verbrannt, auch solche, die wie seine ,Sprach- und Denklehre' schon zum Teil gedruckt waren."14

Dem Theologen Ernst Barnikol ist das Verdienst zuzuschreiben, verschollen geglaubte Dokumente, u.a. aus der Zeit des amerikanischen Exils, während der 1920er Jahre in deutschen Archiven aufgefunden zu haben. Barnikol hat Weitlings Tagebuch aus dem Züricher Gefängnis, Gerechtigkeit. Ein Studium in 500 Tagen, gefunden und publiziert, dazu verschie-

12 „Wilhelm Weitling und sein System der Harmonie und Freiheit", a.a.O., S. 584.

13 Kaler, Wilhelm Weitling, a.a.O., S. 76.

14 Schlüter, Die Anfänge der deutschen Arbeiterbewegung in Amerika, a.a.O., S. 127. 
dene Dokumente über Weitlings Aufenthalt in Hamburg 1848-49.15 Außerdem hat er einige der sprachphilosophischen und astronomischen Studien gefunden und veröffentlicht. ${ }^{16}$ Dabei handelt es sich um den 1848/49 in Hamburg für Alexander von Humboldt angefertigten Extrakt der Weitlingschen Denk- und Sprachlehre, die Klassifikation des Universums; außerdem die erste astronomische Schrift Weitlings, Der bewegende Urstoff in seinen kosmo-electro-magnetischen Wirkungen aus dem Jahr 1856; schließlich um die Theorie des Weltsystems aus dem Jahr 1859. Diese Schriften Weitlings sind längst nicht alle astronomischen Studien Weitlings, aber schon diese von Barnikol gefundene Auswahl ist bislang in der Literatur nicht zur Kenntnis genommen worden. ${ }^{17}$ Die Denk- und Sprachlehre glaubte Barnikol ,verschollen”, die Mechanik des Himmels, Weitlings astronomisches Hauptwerk, wähnte er ,irgendwo in Amerika vorhanden [. . .], falls nicht bereits der Sammeleifer des Moskauer MarxEngels-Institutes bereits diesen Schatz gehoben und dort geborgen hat". ${ }^{18}$

In der europäischen Literatur kaum zur Kenntnis genommen worden ist Carl Wittkes Weitling-Biographie aus dem Jahr $1950 .{ }^{19} \mathrm{Im}$ Vorwort seiner Arbeit dankt Wittke ,first of all [. . .] Terijon Weitling of Staten Island, New York, who so graciously made all the remaining papers of his father available to me and answered all my questions about those personal details which are so important in any biographical study". ${ }^{20}$ Terijon Weitling ist Wilhelm Weitlings 1866 geborener fünfter und jüngster Sohn, das ausführliche Antwortschreiben des achtzigjährigen Terijon Weitling auf Wittkes Fragen hin ist vollständig erhalten. Wittke hat den gesamten Bestand für seine Biographie eingesehen, aber nicht alles Material verwendet. Wahrscheinlich hat Wittke auch für die 1953 erfolgte Übergabe des Nachlasses aus dem Familienbesitz an die Public Library gesorgt; ${ }^{21}$

15 Wilhelm Weitling, Gerechtigkeit. Ein Studium in 500 Tagen, Erstausgabe von Ernst Barnikol, Kiel 1929, Neudruck mit einem Nachwort von Ahlrich Meyer, Berlin 1977; Ernst Barnikol, Weitling der Gefangene und seine „Gerechtigkeit”. Eine kritische Untersuchung über Werk und Wesen des frühsozialistischen Messias, Kiel 1929.

16 Wilhelm Weitling, Klassifikation des Universums, Erstausgabe von Ernst Barnikol, Kiel 1931; ders., Der bewegende Urstoff in seinen kosmo-elektro-magnetischen Wirkungen. Ein Bild des Weltalls, Erstausgabe von Ernst Barnikol, Kiel 1931; ders., Theorie des Weltsystems, Erstausgabe von Ernst Barnikol, Kiel 1931, Zitat S. 14.

17 Zur Interpretation der sprach- und naturphilosophischen Spätschriften Weitlings vgl. Lothar Knatz, Utopie und Wissenschaft im frühen deutschen Sozialismus. Theoriebildung und Wissenschaftsbegriff bei Wilhelm Weitling; erscheint demnächst.

18 Barnikol, Weitling der Gefangene und seine „Gerechtigkeit”, a.a.O., S. 50.

19 Wittke, The Utopian Communist, a.a.O.; vgl. auch ders., ,Wilhelm Weitling's Literary Efforts", in: Monatshefte (Madison), Bd 40 (1948), S. 63-68.

20 Wittke, The Utopian Communist, S. vi-vii.

21 Vgl. Robert W. Hill, ,The Wilhelm Weitling Papers”, in: Bulletin of the New York Public Library, Bd 57 (1953), S. 358-60. 
zweifelsfrei hat er Teile des Bestandes, so wie er heute vorliegt, geordnet. Wittke gibt in seiner Arbeit keine genauen Quellenhinweise, beschreibt in einer bibliographischen Note aber knapp die ihm von Terijon Weitling zur Verfügung gestellten Materialien.

Though Weitling destroyed many of his papers two years before his death, a mass of material survived and was made available to the author by his last surviving son, Terijon Weitling of New York. These manuscripts include correspondence with co-workers in Europe and America, account books of the Arbeiterbund, two notebooks with a miscellany of personal items, and a great many items dealing with the colony of Communia, Iowa, and with Weitling's interest in astronomy, a universal language, and inventions. The Library of Congress also has a few Weitling items. ${ }^{22}$

Wittke bestätigt Schlüters Angabe, wonach Weitling 1869 offenbar einige Manuskripte verbrannt hat. Es sind jedoch weitaus mehr Materialien erhalten geblieben, als Schlüter angenommen hat, so u.a. die Denk- und Sprachlehre und die Mechanik des Himmels. Darüberhinaus nennt Wittke Schlüters Interpretation ,superficial and disappointing". ${ }^{23}$ Bert Andréas haben wir zu danken, daß er den Nachlaß im Rahmen seiner Forschungsinteressen 1973 noch einmal im europäischen Kollegenkreis bekannt gemacht hat; verzeichnet ist der Bestand auch in dem von Philip Hamer herausgegebenen Führer durch die US Archive. ${ }^{24}$

Der New Yorker Weitling-Nachlaß ist der umfangreichste Einzelbestand über Wilhelm Weitling. Er enthält 51 von Weitling geschriebene und 56 an ihn gerichtete Briefe sowie 24 Briefe, die zwischen Dritten gewechselt wurden; handschriftliche und gedruckte Manuskripte Weitlings zu sprachund naturphilosophischen sowie astronomischen Themen: gedruckte Schriftstücke wie die Konstitution der Kolonie Communia und handschriftliche Dokumente wie die Vollmachten für den Arbeiterbund und die technischen Patente; darüberhinaus Geschäfts-, Tage- und Gedichtbücher sowie zahlreiche unzusammenhängende Skizzen und Notizen. Ohne einer gründlichen Auswertung vorgreifen zu wollen, zeichnen sich für die Forschung folgende Schwerpunkte ab.

1. Detaillierte Hinweise über einzelne Phasen der Tätigkeit Weitlings vor 1848.

22 Wittke, The Utopian Communist, S. 317.

23 Ebd., S. 318.

24 Bert Andréas, Notizen aus US-Archiven (1973), Fotokopie im Internationaal Instituut voor Sociale Geschiedenis, S. 9f.; United States National Historical Publications Commission, A Guide to Archives and Manuscripts in the United States, New Haven 1961, S. 25. 
2. Über die Korrespondenz und das Account Book kann die Geschichte der Kolonie Communia erschlossen werden. ${ }^{25}$ Dafür sind über die New Yorker Quellen hinaus die Republik der Arbeiter heranzuziehen sowie lokale Akten und Pressenotizen aus Clayton County, Iowa, wo Communia immer noch als kleine Siedlung existiert und wo immer noch Zeugnisse aus der Zeit als kommunistische Kolonie aufzuspüren sind.

3. Die nähmaschinentechnischen Erfindungen Weitlings und ihre Stellung innerhalb der Technikgeschichte sowie ihre Bedeutung für den geschäftlichen Aufstieg der Firma Singer.

4. Die wissenschaftshistorische Interpretation der Denk- und Sprachlehre und der astronomischen Studien Weitlings, die als Versuche Weitlings gelesen werden können, den Sozialismus von der Utopie zur Wissenschaft zu entwickeln.

Der Nachlaß umfaßt insgesamt 3 Kartons (Boxes). Karton 1 beinhaltet 3 braune Mappen (brown folders) und Karton 2 enthält 5 braune Mappen. Jede braune Mappe ist noch einmal nach weißen Einlegemappen (white folders) untergliedert, in denen sich die nach formalen und inhaltlichen Kriterien grob sortierten Dokumente befinden. Karton 3 ist nicht nach Mappen untergliedert, sondern beinhaltet Schriften und Bücher. In der folgenden Auflistung des Bestandes übernehmen wir die wahrscheinlich von Carl Wittke stammende Ordnung. Wir beginnen bei der Vorstellung der braunen Mappen jeweils mit einem knappen Überblick über deren Inhalt. Die Dokumente sind von uns durchnumeriert worden, um Querverweise zu ermöglichen. Fehlende Daten sind, soweit möglich, in runden Klammern ergänzt. Von uns übernommene Manuskripttitel sind in Anführungszeichen gesetzt. Die Kommentierung will auf uns besonders bemerkenswert erscheinende Dokumente aufmerksam machen und den historischen Kontext einzelner Dokumente erläutern. Wir verzeichnen den Bestand nicht Blatt für blatt, die Briefe haben wir in chronologischer Reihenfolge angeordnet. Notizen und unzusammenhängende Manuskripte erwähnen wir in den Übersichten zu den braunen Mappen, ohne sie anschließend aufzuführen. Inhaltliche Kommentare folgen gegebenenfalls im Anschluß an die Formalbeschreibung nach dem jeweiligen Dokument.

25 Die einzige monographische Arbeit über Communia ist immer noch der Aufsatz von George Schulz-Behrend ,Communia, Iowa. A Nineteenth-Century German-American Utopia", in: Iowa Journal of History, Bd 48 (1950), S. 27-54. 
Karton 1 / 1. braune Mappe

Correspondence 1844-1903; n.d.

In der 1. weißen Mappe findet sich der einzige vor 1850 datierte Brief, dessen Inhalt auf August Becker als Verfasser deutet (Nr 1). August Willich berichtet von der Entlarvung des Spitzels Cherval (Nr 2). Weitlings Kontakt mit Georg Kuhlmann bezieht sich im ersten Brief ( $\mathrm{Nr} 5$ ) auf ein Unterstützungsgesuch für die Familie Scherzer und im zweiten Brief ( $\mathrm{Nr} 8$ ) auf finanzielle Fragen. Ansonsten betreffen die Briefe hauptsächlich den Arbeiterbund und die Kolonie sowie Weitlings astronomische Theorien. Letztere sind vor allem das Thema des ausführlichen Briefwechsels mit Sir Joseph Henry, dem Leiter der Smithsonian Institution in Washington (D.C.); elf Briefe aus der Zeit vom 3. Mai 1856 bis zum 24. August 1866 sind hier überliefert. Ein weiterer Brief Weitlings an Henry vom 13. August 1866 befindet sich im Archiv der Smithsonian Institution.

Die Briefe aus der 2. weißen Mappe behandeln die astronomischen und naturphilosophischen Theorien, dazu auch einige Manuskripte zu diesem Thema (Nr 35-38). Pläne zur Herausgabe der Schriften Weitlings bzw. zur Abfassung von Schriften über ihn werden in insgesamt zehn Briefen behandelt ( $\mathrm{Nr} 30,33,40,45-47,50-53)$. Daraus geht u.a. hervor, daß bereits zu Lebzeiten von Weitling eine Gesamtausgabe seiner Schriften in Deutschland geplant war, die jedoch nie realisiert worden ist $(\mathrm{Nr} 33)$. An Otto Wigand wandte sich Weitling mit der Bitte um Veröffentlichung der Mechanik des Himmels, die dieser jedoch nur übernehmen wollte, wenn Weitling das finanzielle Risiko übernommen hätte ( $\mathrm{Nr} 30)$. Die undatierten Briefe ( $\mathrm{Nr}$ 55-63) betreffen hauptsächlich die Kolonie, so auch der Brief an Albert Brisbane, in dem Weitling um Rat wegen der KolonieStreitigkeiten fragt $(\mathrm{Nr} 60)$.

Die 3. weiße Mappe enthält Fotografien von Briefen, deren Originale in der Library of Congress, Washington (D.C.), aufbewahrt werden. Sie betreffen den Arbeiterbund, die Kolonie und die Republik der Arbeiter. Dabei werden Finanzierungs- und Vertriebsfragen der Zeitschrift, aber auch Redaktionsangelegenheiten erörtert. Verschiedentlich finden sich dabei auch Andeutungen, daß die Republik der Arbeiter die Belange des Arbeiterbundes beschönigend darstelle $(\mathrm{Nr}$ 65, 69). In der 4. weißen Mappe befinden sich ausschließlich Briefe von Friedrich Albert Sorge an Caroline Weitling. Sorge bittet darin um Überlassung von Materialen für schriftstellerische Arbeiten über Wilhelm Weitling; auch Hermann Schlüter wird dabei erwähnt ( $\mathrm{Nr}$ 78-79). Die Briefe aus der 5. weißen Mappe betreffen hauptsächlich die Kolonie und den Arbeiterbund, die undatierten Briefe (Nr 124-4I) auch die Republik der Arbeiter. Hervorzuheben sind die aus- 
führlichen Briefe Otto von Ruedgischs, den Weitling vermutlich 1848 in Berlin kennengelernt hatte und an den er mit der Bitte um Unterstützung herantritt ( $\mathrm{Nr} 117-23){ }^{26}$

\section{1. weiße Mappe: Correspondence 1844-59}

1. (August Becker?) Lausanne, 4. August 1844, an „Mr. Harry” (Weitling), 20 Bloomsbury, High-Street, London, $4 \mathrm{~S}$.

2. August Willich, London, 14. Mai 1852, 2 S., unvollständig, beglaubigt von Stolze.

3. Weitling, Philadelphia, 2. Dezember 1852, an R. Meier, $4 \mathrm{~S}$.

4. (Rechnungsquittung,) 27. Juni 1853, 1 S., englisch, rückseitiger Vermerk "Unwichtige Rechnung in Betreff Arbeiterbund".

5. Georg Kuhlmann, Paris, 2. November 1854, an Weitling, New York, 2 S., mit Bemerkungen Weitlings über Verfahrensweisen, $1 \mathrm{~S}$.

6. Friedrich Röpenack, Hannover, 10. Juli 1855, an Weitling, New York, 3 $\mathrm{S}$.

7. Weitling, New York, 21. Juli 1855, an „Lieben Brüder!”, 3 S.

8. Georg Kuhlmann, Paris, 9. Oktober 1855, an Weitling, New York, 2 S.

9. Kaiserliche Akademie der Wissenschaften, Wien, 20. März 1856, an Weitling, New York, 1 S.; der Brief trägt einen Stempel der Smithsonian Institution, Weitling hat handschriftlich vermerkt ,erhalten 28.2.1857”.

10. Weitling, New York, (März 1856,) an Joseph Henry, Washington, 7 S., englisch.

11. „Report”, $9 \mathrm{~S}$., englisch.

Im Auftrag der Smithsonian Institution angefertigtes Gutachten über Weitlings astronomische Studien.

12. Anonym, Wolga (Communia), 25. April 1856, an Weitling, $3 \mathbf{S}$.

13. Joseph Henry, Smithsonian Institution, Washington, 3. Mai 1856, an Weitling, New York, 2 S., englisch.

14. Weitling, New York, 16. August 1856, an Hofer (Communia), $4 \mathrm{~S}$.

15. Joseph Henry, Smithsonian Institution, Washington, 26. September 1857, an Weitling, New York, 1 S., englisch.

16. Weitling, September 1857, an Joseph Henry, Washington, 4 S., englisch.

17. Hofer, Littleport, 6. Mai 1858, an Weitling (New York), $2 \mathrm{~S}$.

18. John S. Fields, Guttenberg, Iowa, 11. Mai 1858, an Weitling (New York), $1 \mathrm{~S}$., englisch.

Schreiben des Anwaltes von Arnold wegen Kolonie-Streitigkeiten.

19. F. Hofer, Littleport, 4. Juli 1858, an Weitling, New York, $3 \mathrm{~S}$.

20. Weitling, New York, 10. März 1859, an Monsieur le Secrétaire de l'Institut impérial de France, section physicale et mathématique, $12 \mathrm{~S}$., französisch, nicht in Weitlings Handschrift.

21. Prof. Dr A. Ehrenberg, Berlin, 8. April 1859, an Weitling, New York, 1 S. Schreiben der Akademie der Wissenschaften in Berlin.

${ }^{26}$ In Weitlings Adressbuch, das er 1848 in Berlin angelegt hat, findet sich der Name „Otto Riegisch”. Vgl. Staatsarchiv Hamburg, Polizeibehörde Kriminalwesen, C, Serie V, Lit. V, Nr 3072, Bd 3, B1. 25. 
22. Joseph Henry, Smithsonian Institution, Washington, 14. April 1859, an Weitling, New York, 1 S., englisch.

23. Weitling, New York, 24. April 1859, an Joseph Henry, Washington, 2 S., englisch.

24. Joseph Henry, Smithsonian Institution, Washington, 27. April 1859, an Weitling, New York, 2 S., englisch.

25. Kaiserliche Akademie der Wissenschaften, Wien, 16. Juni 1859, an Weitling, New York, $2 \mathrm{~S}$.

26. Weitling, New York, 1859, an Prof. Anton Schrötter, Secretär der Akademie der Wissenschaften zu Wien, 12 S.; Weitling hat am 27. Juli 1864 einen handschriftlichen Nachtrag zu diesem Brief verfaßt.

2. weiße Mappe: Correspondence 1860-1903; n.d.

27. Joseph Henry, Smithsonian Institution, Washington, 26. Oktober 1860 , an Weitling, New York, 1 S., englisch.

28. Ders., Washington, 12. April 1861, an Weitling, New York, 1 S., englisch.

29. Carl Schurz, Camp near Stafford C.H., 21. Februar 1863, an Usher, 1 S., englisch.

Empfehlungsschreiben für Weitling.

30. Otto Wigand, Leipzig, 16. September 1863, an Weitling, New York, 1 S. Der Brief weist außerdem einen Poststempel „Hamburg 17.9.1866” auf.

31. Joseph Henry, Smithsonian Institution, Washington, 24. August 1866, an Weitling, New York, I S., englisch.

32. v. Schleinitz, Berlin, 30. Juni 1868, an Weitling, $3 \mathrm{~S}$.

33. Boruttau, Leipzig, 14. November 1868, an Weitling, New York, 4 S., 2 S. französisch.

34. Astronomisches Manuskript ohne Titel, 3 S., Weitlings Handschrift.

Offenbar handelt es sich um die Seiten 51-53 eines umfangreicheren Manuskriptes.

35. Astronomisches Manuskript ohne Titel, 4 S., Weitlings Handschrift.

36. ,Wilhelm Weitling - Entdeckungen auf dem Felde der theoretischen Astronomie", 4 S., Weitlings Handschrift.

37. „Rechnungsformen, welche ich als Grundlage dieses Systems benutze”, $7 \mathrm{~S}$., astronomisches Manuskript in Weitlings Handschrift.

38. „Die astronomischen Bewegungskräfte”, $28 \mathrm{~S}$., astronomisches Manuskript in Weitlings Handschrift.

39. Weitling, New York, 18. Januar 1870, an M. Hundt, $2 \mathrm{~S}$.

40. J. L. Stiger, Graz, 13. März 1870, an Weitling (New York), 2 S.

41. Ders., Graz, 26. Februar 1871, an die Witwe Weitlings (New York), 4 S.

42. H. W. Rincker, 13. Mai 1873, an die Witwe Weitlings (New York), 2 S., englisch.

43. Unvollständige Liste der Schriften Weitlings sowie einiger anderer Titel, darunter u.a. Stiger, ,An die Arbeiter Amerikas", 2 S.

44. (Friedrich) Toedt, Ithaca, 11. Februar 1876, an "Liebe Tochter!" (Caroline Toedt, Weitlings Witwe, New York), 3 S.

45. J. L. Stiger, Zürich, 10. September 1877, an die Witwe Weitlings (New York), $4 \mathrm{~S}$. 
46. Ders., Zürich, 5. April 1878, an die Witwe Weitlings, $4 \mathrm{~S}$.

47. Ders., Zürich, 26. Juli 1878, an die Witwe Weitlings (New York), Postkarte.

48. „An die Arbeiter Amerikas”, mit einer Widmung „Der Frau Witwe Karoline Weitling zur freundlichen Erinnerung. Zürich am 18. Oktober 1878", von J. L. Stiger, 4 S., gedruckt.

49. Zettel mit der Adresse von Dr C. Hugo, 1895, 1 S.

50. M. Ernst, München, 30. Mai 1901, an Hugo Lindemann, 3 S.

51. Hermann Schlüter, New York, 9. Februar 1903, an Wilhelm Weitling jun., New York, 2 S., Briefkopf der New Yorker Volkszeitung, beiliegend eine Visitenkarte von Hermann Schlüter.

52. Wilhelm Weitling jun. (New York), 9. Mai 1903, an Hermann Schlüter (New York), $2 \mathrm{~S}$.

53. Hermann Schlüter, New York, 14. Mai 1903, an Wilhelm Weitling jun., New York, I S., Briefkopf der New Yorker Volkszeitung.

54. Briefumschlag, adressiert an Frau Witwe Wilhelm Weitling, 178 Stanton Street, New York, aus Wien.

55. Mühle bei Garnavillo, 30. Juni, 2 S.

Protokoll von Weitling über Verhandlungen mit Henry Barnhart über den Bau einer Mühle in der Nähe der Kolonie Communia.

56. Weitling, Baltimore, 11. März, an die Republik der Arbeiter, $1 \mathrm{~S}$.

57. Ders. an Unger, $2 \mathrm{~S}$.

58. Ders. an den New York Demokrat, 1 S., rückseitig Notizen zur Denk- und Sprachlehre.

59. Ders. an James Burt, Dubuque, I S., englisch.

60. Ders. an Albert Brisbane, 2 S., englisch.

61. Ders. (New York) an Michel Chevalier, Institut de France, Section astronomique, $2 \mathrm{~S}$., französisch.

Entwurf für Nr 63.

62. August Witzleben, Berlin, 10. August, an Weitling, $4 \mathrm{~S}$.

63. Weitling (New York) an Membres de l'Institut de France, Section des sciences mathématique et physicales, $4 \mathrm{~S}$., französisch.

3. weiße Mappe: Correspondence, Photostats, 1851; n.d.

64. Weitling, New York, 6. Januar 1851, an ,Lieben Brüder!”, $4 \mathrm{~S}$.

65. Ders., New York, 18. Februar 1851, $8 \mathrm{~S}$.

Kreisschreiben an die Tauschkommissionen und Centraltauschkommissionen der Verbrüderung.

66. Ders., New York, 29. April 1851, an Johann Krieg, Cincinnati, 1 S.

67. Ders., New York, 15. Mai (1851), an „Lieben Brüder!”, 2 S.

An die Kolonisten in Communia gerichtet.

68. Ders., New York, 23. Juni 1851, an Brüder in Cincinnati, $1 \mathrm{~S}$.

69. Ders., New York, 25. Juni 1851, an (Johann) Krieg, 3 S.

70. Ders., Communia, 3. Oktober 1851, an (Johann) Krieg, Cincinnati, $3 \mathrm{~S}$.

71. Ders., New Orleans, 20. Januar (1852), an (Johann) Krieg, 1 S.

72. Ders., Communia, 9. Juli 1852, an (Johann) Krieg, 4 S. 
73. Ders., Detroit, 26. August (1852), an (Johann) Krieg, $1 \mathrm{~S}$.

4. weiße Mappe: Correspondence: Letters from Friedrich Adolph Sorge to Mrs Weitling, 1895, 1896, 1902

74. Friedrich Adolph Sorge, Hoboken, 29. September 1895, an Frau Weitling (New York), $3 \mathrm{~S}$.

75. Ders., Hoboken, 23. März 1896, an Frau Weitling (New York), 4 S.

76. Ders. und Frau, Hoboken, 3. Mai 1896, an Frau Weitling (New York), $1 \mathrm{~S}$.

77. Ders. und Frau, Hoboken, 17. Mai 1896, an Frau Weitling (New York), $2 \mathrm{~S}$.

78. Ders. und Frau, Hoboken, 1. Juli 1896, an Frau Weitling (New York), $3 \mathrm{~S}$.

79. Ders. und Frau, Hoboken, 22. Oktober 1902, an Frau Weitling (New York), $1 \mathrm{~S}$.

5. weiße Mappe: Correspondence relating to the Communia Working-Mens League (Arbeiterbund) Colonies 1852-69; n.d.

80. Weitling, Natchez, 7. Februar 1852, an Republik der Arbeiter, 2 S.

81. Ders., Natchez, 7. Februar 1852, an Republik der Arbeiter, 2 S., Vermerk von Weitling ,späterer Brief von hier, d.i. zweiter”.

82. Rudolph Kreter, 25. Mai 1852, an Weitling, New York, 1 S.

Quittung für die Kolonie Communia über \$ 1924,30.

83. Weitling, Communia, 20. Juli 1852, an „Lieben Freunde!”, 4 S.

84. Ders., Communia, 21. Juli 1852, an die Bundesgemeinde (des Arbeiterbundes) in St Louis, $12 \mathrm{~S}$., unvollständig.

85. Central-Kommission des Arbeiterbundes, New York, 24. Oktober 1852, an Simon Schmidt, Kalamazoo, 4 S., nicht in Weitlings Handschrift.

86. New York, 2. November 1852, an die Mitglieder der Kolonie Communia, $2 \mathrm{~S}$., nicht in Weitlings Handschrift.

87. Weitling, Communia, 1. Januar 1853, an Rudolph Kreter, 4 S.

88. J. M. Baumann, Forklanding, 20. Februar 1853, an Weitling, $2 \mathrm{~S}$.

89. Ders., Forklanding, 2. Mai 1853, an Weitling, $2 \mathrm{~S}$.

90. Weitling, Communia, 5. Juli 1853, an die Central-Commissionen, New York, $1 \mathrm{~S}$.

91. Ders., Communia, 14. Juli 1853, an die Central-Commissionen, New York, $12 \mathrm{~S}$.

92. Ders., Communia, 22. Juli 1853, an Republik der Arbeiter, $4 \mathrm{~S}$.

Editorielle Korrespondenz.

93. „25. August 1853 bis 1850 Communia. Colony Community. Communia Association. Association: Communia-Workingmens-League", $4 \mathrm{~S}$.

Bericht an die Kolonie Communia über die Verabschiedung einer neuen Verfassung; merkwürdige Datierung.

94. Weitling, Communia, 23. Dezember 1853, an (Rudolph) Kreter, $4 \mathrm{~S}$.

95. Elias H. Williams, Attorney of the Communia Working Mens League, 12. Januar 1854, an Weitling, 3 S., mit Randnotizen von Weitling. 
96. Henry Barnhart, Millwright, Communia, 30. März 1854, an Weitling, New York, 2 S., englisch.

97. Weitling, 4. April 1854, 2 S. deutsch und $1 \mathrm{~S}$. englisch. In Weitlings Handschrift ein ,Instructions-Bericht vor Abgang in die Kolonie”, dazu eine Erklärung von Weitling für den Fall seines Todes.

98. J. F. Klopfer, Communia, 26. Mai 1854, an Justus Metke, Keokuk, Iowa, $1 \mathrm{~S}$.

99. Henry Barnhart, Littleport, 30. Mai 1854, an Weitling, New York, 2 S., englisch.

100. James Burt, Dubuque, 12. Juli 1854, an Weitling, 4 S., englisch.

101. Ders., Dubuque, 22. August 1854, an Weitling, 3 S., englisch.

102. F. Hofer, Dubuque, 1. Juni 1856, an Weitling, $2 \mathrm{~S}$.

103. Ders., Dubuque, 10. August 1856, an Weitling, New York, 1 S.

104. Denis Gugley, Littleport, 10. Oktober 1856, an Weitling, 2 S., englisch; Gugley schreibt ,Whiteling”.

105. F. Hofer, Dubuque, 15. Oktober 1856, an Weitling (New York), $2 \mathrm{~S}$.

106. Ders., Dubuque, 9. November 1856, an Weitling (New York), 1 S.

107. Ders., Wolga, 12. April 1857, an Weitling (New York), 2 S.

108. Ders., Dubuque, 30. Mai (1857), an Weitling (New York), 2 S.

109. Ders., Littleport, 11. August 1858, an Weitling, New York, 1 S.

110. F. Hagen und L. Brandenberger, Communia, 26. September 1858, an „Lieben Brüder!', $2 \mathrm{~S}$.

111. B. F. Weis, Communia, 26. September 1858, an Weitling, $1 \mathrm{~S}$.

112. N. Peters, Cincinnati, 4 . November 1858, an Weitling, $2 \mathrm{~S}$.

113. Henry M. Jones, Littleport, 15. November 1858, an Weitling, New York, 2 S., englisch.

114. F. Hofer, Littleport, 16. November 1858, an Weitling, New York, 1 S., zusammen mit $\mathrm{Nr} 113$ in einem Briefumschlag.

115. B. F. Weis, Communia, 8. Juli 1860, an Weitling (New York), $1 \mathrm{~S}$.

116. August Heimerdinger, Louisville, 1. November 1864, an Weitling (New York), I S.

117. Otto von Ruedgisch, Newton, Illinois, 20. April 1868, an Weitling (New York), $2 \mathrm{~S}$.

118. Ders., Newton, 10. Juli 1868, an Weitling (New York), 2 S.

119. Ders., Newton, 1. August 1868, an Weitling (New York), 4 S.

120. Ders., Newton, 26. August 1868, an Weitling (New York), $4 \mathrm{~S}$.

121. Ders., Newton, 13. September 1868, an Weitling (New York), 2 S.

122. Ders., Newton, 9. August 1869, an Weitling (New York), 2 S.

123. Ders., Newton, 24. August 1869, an Weitling (New York), 3 S.

124. Weitling, Peoria, 22. August, an Republik der Arbeiter, $3 \mathrm{~S}$.

125. Ders., Dubuque, 27. Juli, 4 S., überschrieben ,Thatsachen”.

126. Ders., Peoria, 21. August, an Republik der Arbeiter, 1 S.

127. Ders., an „Lieben Freunde!”, $4 \mathrm{~S}$.

128. Ders., $4 \mathrm{~S}$., überschrieben ,Privatim".

129. Ders., Dubuque, 19. Juli, an Central-Commissionen, New York, $1 \mathrm{~S}$.

130. Ders., Garnoville, Juni, an Central-Commissionen, New York, 1 S.

131. Ders., St Louis, 17. August, an „Lieben Brüder!”, 4 S. 
132. Ders., Communia, an „Lieben Brüder!”, $4 \mathrm{~S}$.

133. Ders., St Louis, 18. August, an Republik der Arbeiter, 1 S., unvollständig.

134. Ders., Iowa, 13. Juni, an Republik der Arbeiter, 3 S.

135. Ders., Natchez, 10. Februar, an Republik der Arbeiter, 2 S.

136. Frau Brandenberger, Carington (?), 17. Juli, an Weitling, $1 \mathrm{~S}$.

137. Weitling, Baton Rouge, 3. Februar, an Republik der Arbeiter, $1 \mathrm{~S}$.

138. Ders., St Louis, 16. August (1853?), an Republik der Arbeiter, 1 S., Anrede „Lieber.Kreter!”

139. Ders., Communia, 22. Juni, an Republik der Arbeiter, 3 S. Editorielle Korrespondenz.

140. Ders., Cincinnati, 5. Juni, an Republik der Arbeiter, I S.

141. Ders. an „Lieben Freunde!”, 2 S., überschrieben „Privatim”.

Karton 1 / 2. braune Mappe

Papers relating to the Communia Working-Mens League (Arbeiterbund) Colonies

Die Dokumente in der 1 . weißen Mappe bevollmächtigten Weitling als Vertreter des Arbeiterbundes. Eine Vielzahl von Mitgliedern des Arbeiterbundes werden hier als Vollmachtgeber auch namentlich bekannt. Die 2. und 3. weiße Mappe enthalten Konstitutionen der Kolonie und des Arbeiterbundes. Während die handschriftliche Konstitution in der 2. weißen Mappe offenbar ausschließlich die Belange der Kolonie betrifft, dokumentieren die gedruckten Fassungen in der 3. weißen Mappe den Versuch, Kolonie und Arbeiterbund enger miteinander zu verbinden. Auch die Dokumente dieser Mappen sind zum Teil namentlich unterzeichnet. Die Skizze in der 4. weißen Mappe zeigt auf der einen Seite die Lage der geplanten Wassermühle am Wolga-River, auf der Rückseite die Verteilung der Grundstücke der Kolonien Communia und Liberty. Die Dokumente der 5. weißen Mappe betreffen sehr verschiedene Belange der Kolonie sowie des Arbeiterbundes ohne übergreifendes Thema, wir haben eine Auswahl aufgelistet.

1. weiße Mappe: Documents appointing Weitling to act as attorney for the Working-Mens League, 1854, 1855

142. Weitling, ,Remarks”, I S., englisch.

143. Vollmacht der Gemeinde in Cincinnati, 5 S., englisch.

144. Vollmacht aus New Orleans, 14. März 1854, 3 S., englisch.

145. Vollmacht aus Philadelphia, 1 S., englisch.

146. Vollmacht aus Cleveland, Ohio, $1 \mathrm{~S}$., englisch.

147. Vollmacht, 1854, 1 S., englisch.

148. Vollmacht, State of Michigan, County of Wayne.

149. Vollmacht, State of Maryland, City of Baltimore, 17. März 1854.

150. Vollmacht, County of Jefferson, State of Kentucky, 14. März 1854. 
151. Vollmacht, State of New Jersey, 28. März 1854.

152. Vollmacht, State of Missouri, County of St Louis, 8. März 1854.

153. Vollmacht.

2. weiße Mappe: Rules of the Communia, Iowa, Colony, April 29, 1853

154. Organisationsgesetze der Kolonie Communia, 29. April 1853, Handschrift, 20 Paragraphen, 4 S., von 20 Personen unterschrieben.

3. weiße Mappe: Constitutions, 1853

155. „Constitution of the Association called Communia Working-Men's League of Clayton County, Iowa", Garnavillo, Clayton County Herald Print, 1853, gedruckt, 60 Paragraphen, 7 S., unterzeichnet von 22 Personen.

156. Dasselbe.

157. „Beschluss der Central-Commission über die Organisation der verschiedenen Schops-Präsidenten", New York, Buchdruckerei von John Weber, 58 Chatham Street, 1850, gedruckt, 8 Paragraphen, 6 S., mit Stempelaufdruck ,Taylors Protective Union - New York”.

158. „The Workingmens-League nebst der Konstitution des Bauvereins des Arbeiterbundes der Kolonie Communia", New York, Druckerei der „Republik der Arbeiter”, 1853, gedruckt, 15 S.; 8 S. „The Workingmens-League" (deutsch), 24 Paragraphen, $5 \mathrm{~S}$. Konstitution.

159. Clayton County Herald (Garnavillo, Iowa), August 5, 1853, Artiket auf S. 1 "Constitution of the Association called Communia Working Men's League, of Clayton County, Iowa. Resolution of the Undersigned", textgleich mit $\mathrm{Nr}$ 155-56.

4. weiße Mappe: Plan for a water-mill [?]. Verso: Map of the Colonies of Communia and Liberty in Iowa

160. Lageplan und Skizzen der geplanten Wassermühle am Wolgariver. Rückseite: Lageplan der Kolonien Communia und Liberty, Township N 92 Range 4 West.

5. weiße Mappe: Miscellaneous Papers

161. B. F. Weis, Protokoll vom 28. März 1853, 2 S.

162. Map Colony Communia.

163. Colony, 8. Juni 1853, Deed of Trust for Wilhelm Weitling.

164. Communia, July 1852, Deed of Trust for Wilhelm Weitling.

165. Gerichtsvorladung für Weitling in Sachen Communia für den 22. Mai 1854 nach Garnavillo.

166. Zwei Namenslisten von Mitgliedern der Working-Men's League, 1852, 3 $\mathrm{S}$.

167. Namenslisten von Mitgliedern der Working-Men's League, geordnet nach Städten: Philadelphia, Cleveland, New Orleans, Cincinnati, St Louis, Baltimore. 
168. Editorielle Korrespondenzen, $1 \mathrm{~S}$.

169. Inventar-Verzeichnis der Kolonie Communia, 5. Juli 1853, Weitlings Handschrift, $3 \mathrm{~S}$.

170. Jacob Ponsar and Benjamin F. Weis vs. The Working-Men's League, Rudolph Kreter and others, District Court Clayton County, 13 S.

171. Editorielle Korrespondenz, 13. Mai 1853, 1 S.

172. „Indenture, July 1853, between Colony Communia of Clayton County and Communia Association".

Karton 1/3. braune Mappe

Sewing Machine Patents and Plans

Lose eingelegt sind sieben großformatige Nähmaschinen-Patente einschließlich technischer Zeichnungen und dazugehöriger Erläuterungen. Wie aus seinem Tagebuch hervorgeht, hat Weitling 1855 erstmals eine Eingabe an das Patent Department in Washington gemacht, die ebenso wie nachfolgende Eingaben abschlägig beschieden worden ist. Das erste Patent datiert vom 29. Oktober 1861. Offenbar hat Weitling auch beträchtliche Summen entliehen und investiert, um die Funktionstüchtigkeit seiner Entwürfe im Modell nachweisen zu können. Zunächst hat er große Hoffnungen darauf gesetzt, sich über den Verkauf der Patente finanziell sanieren zu können, mit seinem wertvollsten Patent wurde er jedoch von dem Nähmaschinenfabrikanten Singer betrogen. In der New Yorker Volkszeitung vom 28. März 1907 (vgl. Nr 245) wird berichtet, daß Singer und seine Techniker sich die Knopfloch-Stickmaschine von Weitling vorführen ließen und das Patent anschließend nachbauten. Weitling fehlten die finanziellen Mittel, um den folgenden Prozeß durchstehen zu können. Für den Theoriebildungsproze $\beta$ des Handwerksgesellen und Autodidakten ist sicherlich bedeutsam, daß er mehr als ein Jahrzehnt, abgeschnitten von der Möglichkeit politisch-praktischer Betätigung, neben der Verpflichtung des täglichen Broterwerbs nur an zwei Dingen wirkliches Interesse hatte: den Nähmaschinen-Konstruktionen und den Konstruktionen der „Mechanik des Himmels”. Für Weitling waren nicht nur die Konstruktionsprinzipien der handwerklich-praktischen und der wissenschaftlich-theoretischen Arbeit die gleichen, er war auch in beiden Fällen von der überragenden Bedeutung seiner Arbeiten überzeugt. Im Tagebuch notiert er unter dem Datum vom 7. Juni 1866: „Ich war nie so vollkommen überzeugt von der Superiorität meiner Maschine."

Die 1. weiße Mappe enthält Zeichnungen und technische Erläuterungen dazu, die 2. weiße Mappe mit einer Ausnahme ( $\mathrm{Nr} 181$ ) Dokumente, die im Zusammenhang mit den Verbesserungen an der Nähmaschine stehen. Darunter findet sich ein Brief des Fourieristen Charles Dana an Weitling, 
aus dem hervorgeht, daß Weitling jenen um finanzielle Unterstützung für seine Nähmaschinen-Patente gebeten hat. Dana schließt: „I wish I could help you, but I can't."

Lose Einlage: 7 großformatige Patente für Wilhelm Weitling in New York

173. Improvement in Sewing Machines, 24. Dezember 1867.

174. Improvement in Sewing Machines, 3. Januar 1865.

175. Improvement in Device for Equalizing the Delivery of Thread from Shuttles and Spools of Sewing Machines, 21. Dezember 1865.

176. Improvement in Tension-device for Sewing Machine Shuttles, 10. Mai 1864.

177. Improvement in Sewing Machines, 17. März 1863.

178. Improvement Stitch for Button holes, 18. Februar 1862.

179. Improvement in Sewing Machines, 29. Oktober 1861.

1. weiße Mappe: Plans for Improvements to the Sewing Machine

180. 7 Blätter mit Zeichnungen zu den Erfindungen.

2. weiße Mappe, ohne Titel

181. Zeitungsausschnitt aus dem Philadelphia Tageblatt, 1. November 1895. Aufruf nach Weitlings Schriften für eine Gesamtausgabe im Verlag M. Ernst, München, für die Dr C. Hugo eine Einleitung verfassen soll.

182. E. Koehler, Baltimore, 26. Juli 1863, an Weitling, 2 S.

183. Charles Dana, Chicago, 30. Dezember 1865, an Weitling, I S., englisch.

184. Agreement between William Weitling and James N. Phelps, rel. Sewing Machine Inventions, 1. Juni 1865, H. E. Famsworth, Counsellor at Law, 133 Nassau Street, New York, 8 S., englisch.

185. U.S. Patent Office, Washington, 16. Mai 1868, an Weitling, 2 S., englisch.

186. Official Gazette of the United States Patent Office, Bd 2, Nr 11, 27. August 1872.

187. Official Gazette of the United States Patent Office, $\mathrm{Bd} 2, \mathrm{Nr} 9,13$. August 1872, S. 223-24: „Commissioner's Decisions. W. Weitling, H. Sauerbier and A. J. Cross vs. S. G. Cabell et al - Interference".

188. J. Clayton, 7. Dezember 1868, an Weitling, 2 S., englisch.

189. Weitling ,To the Commissioner of Patents”, Eingangsstempel 27. Mai $1868,9 \mathrm{~S}$.

190. J. Clayton, 11. Dezember 1868, an Weitling, 2 S., englisch.

191. Weitlings Stickerei-Maschine, Beilage zur Zeichnung, Stellung der Maschine für Stiche 1 und 2, $1 \mathrm{~S}$.

192. "Weitlings Embroidery Machine. Specifications to Sketches”, $2 \mathrm{~S}$.

193. „Treatise on Sewing-machines generally and on the arrangements for sewing by it buttonholes and embroidering specially", $8 \mathrm{~S}$. 
Karton 2 / 1. braune Mappe

Writings, Astronomical Papers

Der Inhalt der beiden weißen Mappen ist nur teilweise geordnet. Insgesamt handelt es sich um ca 324 Bogen und Blätter mit astronomischen Studien, Zeichnungen und Berechnungen. Vieles hat den Charakter von Notizen. Einige Entwürfe sind mehrfach vorhanden, entweder mehrfach deutsch in Weitlings Handschrift oder in zusätzlich französischer oder englischer Übersetzung deutschsprachiger Vorlagen, wobei die Übersetzungen jeweils nicht von Weitling selbst stammen. Die nicht von Weitling geschriebenen fremdsprachigen Manuskripte sind offenbar als wissenschaftliche Korrespondenz bestimmt gewesen, darunter der handschriftliche Entwurf der Theorie des Weltsystems aus dem Jahr 1859. Weitling hat meist deutsch geschrieben, ausnahmsweise auch in nicht ganz flüssigem und fehlerfreiem Englisch. Die Datierung der einzelnen Manuskripte ist, soweit sie nicht unmittelbar aus dem Text hervorgeht, schwierig. Das früheste datierbare Manuskript stammt vom 29. Mai 1859, das späteste vom 18. September 1870. Einigen wenigen Manuskripten hat Weitling in seiner Altersschrift, möglicherweise während der schweren Krankheit 1869, einen erläuternden Kommentar hinzugefügt. Diese Zusätze sind offenbar für die Nachwelt gedacht gewesen; sie zeigen Weitlings Vertrauen in den Wert seiner Studien, zugleich die Resignation, zu Lebzeiten keine Anerkennung mehr zu erfahren.

Wir haben auf eine Auflistung Blatt für Blatt verzichtet und im folgenden jene Manuskripte aufgeführt, die durch Seitennumerierung oder inhaltlich erkennbar als zusammenhängend identifiziert werden konnten und die jeweils eine eigene Überschrift tragen. Einige dieser Manuskripte sind selbstständige Studien, andere zwar in sich geschlossen, aber offenkundig, etwa durch die Seitennumerierung, Teile eines umfangreicheren Ganzen. Die Aufzählung folgt der Anordnung im Karton und ist nicht identisch mit der Reihenfolge der Entstehung. Weitlings astronomisches Hauptwerk, die Mechanik des Himmels, ist hier nicht den Astronomical Papers zugeordnet. Im Charakter der Studien fällt auf, daß Weitling weder einen fachwissenschaftlichen noch einen disziplinären Zugang zu seinen Themen gewinnt, sondern sich gern in übergreifenden Weltbildzusammenhängen äußert. Genau wie in den gesellschaftstheoretischen Schriften der dreißiger und vierziger Jahre sieht sich Weitling auch in der Astronomie im Kampf gegen mächtige Dogmen in der Wissenschaft und gegenüber Wissenschaftlern, denen soziale Interessen über Erkenntnisinteressen gehen. 
1. weiße Mappe: Astronomical Papers, 1 of 2 Folders

194. „Problem. Angenommen die magnetischen Pole der Erde seien ihre wirklichen Rotationspole, welches muß dann das Gesetz und System der Bewegung der himmlischen Körper sein das sowohl der Deklination der Sonne als allen bestätigten Beobachtungen dieser Bewegung entspricht", 1 Bogen, nachträglicher Vermerk „By Wilhelm Weitling”.

Nr 197 enthält die vollständigere englische Fassung dieses Textes.

195. ,The mysteries of astronomy solved by William Weitling”, $16 \mathrm{~S}$., Vermerk in Weitlings Handschrift ,Geschrieben etwa 6 Monate bevor ich Castle Garden verlassen. Jos. Purdys Freund eine Copie gegeben. (Mr. Olsen)."

Weitling hat die Anstellung in Castle Garden am 11. Juni 1862 aufgegeben, demnach ist der Text um die Jahreswende 1861-62 entstanden. Der Text ist identisch mit $\mathrm{Nr} 200$.

196. „Wm. Weitlings Astronomische Studien. Il. Abschrift”, 42 S.

Die Seiten sind durchnumeriert und umfassen die Gliederungspunkte 75-99, waren also offenbar Teil eines umfangreicheren Textes. Auf der Außenseite der Einbandmappe befindet sich ein später von Weitling zur Erläuterung hinzugefügter Text, aus dem hervorgeht, daß dieser Text vor der Mechanik des Himmels entstanden ist. Das Manuskript existiert in einem zweiten, jedoch weniger vollständigen Exemplar.

197. „Problem: Supposing the magnetical poles of the earth to be its real poles of rotation, find the system which accounts for the Declination of the sun and corresponds with all the observations of the motion of the heavenly bodies", $11 \mathrm{~S}$. auf 3 Bogen.

Vgl. Nr 194.

198. „A few truths which I have discovered and can fully proove”, 4 S., 6 Gliederungspunkte.

2. weiße Mappe: Astronomical Papers, 2 of 2 Folders

199. „A mechanical device showing the true motions of the Sun and Earth", I S., Manuskript bricht ab.

200. „The mysteries of astronomy solved by William Weitling”, $16 \mathrm{~S}$.

Vgl. Nr 195.

201. „Folgendes Schreiben enthält Argumente zu Gunsten der Theorie, welche ich in der Schrift ,Der bewegende Urstoff in seinen kosmoelektro-magnetischen Wirkungen' entwickelte. New York, 29.5.1859, Wilhelm Weitling, Nr. 195 Av. B", 15 S.

Bei diesem Briefentwurf handelt es sich um die Theorie des Weltsy. stems.

202. „Widersprüche der astronomischen Himmelsmechanik mit unsern Elementar Begriffen mechanischer Gesetze", 28 S., mit Bindfaden zusammengebundenes Manuskript.

203. Ohne Titel, folgende Gliederung: I. Die Gestalt unserer Erde; II. Die tägliche Bewegung der Sterne; III. Die tägliche Bewegung der Sonne mit Bezug auf die Sterne; IV. Die Bahnen der Sonne und Erde von den Polen 
des Himmels gesehen; V. Das Vorrücken der Nachtgleichen, 22 S., mit Bindfaden zusammengebunden, einseitig beschrieben, auf der Rückseite mitunter Berechnungen.

Es handelt sich um einen Entwurf für die Mechanik des Himmels, vgl. $\mathrm{Nr} 213$.

204. „Mechanisches und arithmetisches Skeleton meines Systems”, 22 S. (S. 8-29), mit Bindfaden zusammengebunden, einseitig beschrieben, rückseitig manchmal Berechnungen.

Karton 2 / 2. braune Mappe

Mineralogy; Universal Language; Miscellany

In dieser Mappe finden sich Manuskripte zur Denk-und Sprachlehre und der Klassifikation des Universums, darunter aber auch in der 3. weißen Mappe die Mechanik des Himmels, die Weitling in einem Brief an Schilling vom 22. Juli 1869 als ,das wertvollste Buch, das je in der Welt erschienen ist und erscheinen wird", bezeichnet hat ${ }^{27}$ Die Astronomie ist in Weitlings Konzeption einer Universalwissenschaft ein Bestandteil derselben, das Manuskript müßte also richtiger den Astronomical Papers der 1. braunen Mappe zugeordnet werden. Das Manuskript scheint abgeschlossen zu sein, es umfaßt in Weitlings Handschrift 72 Seiten Text und 13 Seiten Zeichnungen mit Erläuterungen. In der Mechanik des Himmels kämpft Weitling gegen das kopernikanische Weltsystem; er ist davon überzeugt, Jahrhunderte alte Irrtümer korrigiert zu haben. Die Einleitung beginnt mit dem Satz: „Vorliegende Schrift wird dem Leser, dem Astronomen wie dem Layen, eine Leuchte sein in der geistigen Finsterniss der modernen astronomischen Wissenschaft.” Im 8. Kapitel, überschrieben „Was mich zum Nachdenken über die Mechanik des Himmels gebracht", beschreibt Weitling die Entstehungsgeschichte der Schrift und erläutert den $\mathrm{Zu}$ sammenhang von Denk- und Sprachlehre und Astronomie. Er kam 1854 während der Arbeit an der Denk- und Sprachlehre auf das Problem, die Begriffe in allen Wissenschaften klassifizieren zu wollen und stieß dabei in der Astronomie auf viele ungelöste Fragen, die ihn fortan immer mehr fesselten und von seinem Ausgangspunkt entfernten.

Die 1. weiße Mappe enthält ein Manuskript, dessen Klassifikationsbeispiel in der Reihenfolge Farben - Pflanzen - Minerale dem Aufbau der Klassifikation des Universums entspricht. Die 2. weiße Mappe enthält Skizzen, die unmittelbar zur Denk- und Sprachlehre gehören, insgesamt ca 119 Seiten. Auch die 4. weiße Mappe enthält Notizen zur Klassifikation und zur Denk- und Sprachlehre, vor allem aber das nach der Mechanik des Himmels umfangreichste geschlossene naturphilosophische Manuskript

27 Schlüter, Die Anfänge der deutschen Arbeiterbewegung in Amerika, S. 126. 
Weitlings, Die Größe der Erde. Es geht darin um den Nachweis, daß die astronomische Refraktionslehre ein Irrtum ist.

1. weiße Mappe: Mineralogy

205. „Kapitel B: Klassifikationsbeispiele. Das Thierreich”, 14 S., durchgehend numeriert von S. 9 bis S. 22.

Es folgen die Gliederungspunkte Farben, Pflanzenreich und Mineralreich; abweichend zur Uberschrift fehlt die Klassifikation des Tierreichs. Das Manuskript ist nicht text-, aber sinnidentisch mit Nr 215.

2. weiße Mappe: Universal Language Sketches

206. „VIII Komposition der Klassenzeichen”, 4 S.

207. "Klassenvertheilung”, 2 S., Schema.

208. „Verbindungsregel”, $4 \mathrm{~S}$.

209. „Körperbegriffe”, $10 \mathrm{~S}$.

210. „Innere Gefühlswirkungen”, $8 \mathrm{~S}$.

211. "Allgemeine Ubersicht der Klassifikations-Verbindungen”, $8 \mathrm{~S}$.

212. Ohne Titel, 44 S. (S. 264-312), Überschrift auf S. 271 „Die Komposition der Klassenzeichen. A. Das Alphabet".

3. weiße Mappe: Miscellany

213. „Die Mechanik des Himmels”, 85 S., einseitig beschrieben und durchgehend numeriert, späterer Zusatz von Wilhelm oder Terijon Weitling „Von Wilhelm Weitling, in his handwriting”.

4. weiße Mappe: Miscellany, German Gothic Script

214. „Die Größe der Erde”, 68 S., einseitig beschrieben, nachträglicher Zusatz „By Wilhelm Weitling in his own handwriting".

215. „C. Repräsentation der Klassifikation der Pflanzen”, 4 S.

$\mathrm{Vgl}$. Nr 205; der Text findet sich ein weiteres Mal in dieser Mappe.

Karton 2 / 3. braune Mappe

Poetry; Play; Will of John F. Bard

Die Gedichte in der 1 . weißen Mappe stammen der Handschrift nach wahrscheinlich nicht von Weitling. Das Schriftbild der ersten beiden Hefte ist jeweils einheitlich, dagegen scheinen die Gedichte im dritten Heft von verschiedenen Personen geschrieben zu sein. Von den drei Heften scheint nur das zweite vollständig zu sein; das letzte Gedicht in Heft 1 bricht unvermittelt $a b$, während das erste Gedicht in Heft 3 mitten in der vierten Strophe beginnt. Thematisch behandeln die Gedichte des ersten Heftes die Revolution von 1848-49 und dürften auch aus dieser Zeit stammen. ${ }^{28} \mathrm{Die}$

28 Vgl. Wittke, The Utopian Communist, S. 126, wo diese Gedichte kurz erwähnt sind. 
Gedichte des zweiten Heftes sind jeweils mit einer Jahreszahl versehen und entstanden demnach in den Jahren 1842-44. Das dritte Heft enthält Liebesgedichte ohne Hinweis auf die Entstehungszeit. Sofern sich die Verfasser der Gedichte ermitteln lassen (was insbesondere bei Heft 2 aussichtsreich erscheint), dürften sich wertvolle Hinweise auf die persönlichen Verbindungen Weitlings während des Vormärz und der 48er Revolution ergeben.

Die Herkunft des Theaterstückes in der 2. weißen Mappe wird durch einen Brief Friedrich Röpenacks aufgeklärt (vgl. Nr 6). Um die Mittel für die Überfahrt nach Amerika zusammenzubekommen, bat Röpenack Weitling, den Vertrieb seiner Theaterstücke in den USA zu übernehmen. Ein eventueller Überschuß sollte an Communia gehen, wo auch Röpenack seine letzten Tage beschließen wollte. Auf dem Titelblatt ist „B. Siegel” als Verfasser genannt, wobei es sich wahrscheinlich um ein Pseudonym Röpenacks handelt. Ein weiteres Stück Röpenacks, der Schwank Der Sylvesterschmaus, wurde in der Republik der Arbeiter vom 15. Juli 1854 abgedruckt. Die Bedeutung des Testaments in der 3. weißen Mappe läßt sich vorläufig nicht klären. Über John F. Bard ist bisher nichts weiter bekannt, als $\mathrm{da} B$ er aus Philadelphia stammte.

\section{1. weiße Mappe: Poetry}

216. Gedichtheft, $8 \mathrm{~S}$., mit folgenden Gedichten: ,Es gilt noch einen Kampf auf Tod u Leben”, ,Hilf Dir selbst”, ,Kampfgesang”, ,Berlin den 25. November" (neben dem Titel notiert „,Berlin 25/11.48”), ,Der Invalide” (bricht ab am Ende der Seite).

217. Gedichtheft, $20 \mathrm{~S}$., davon S. 1-11 beschrieben, mit folgenden Gedichten (Jahreszahlen und andere Zusätze befinden sich immer am Ende der Gedichte und werden hier in Klammern angefügt): „Die Rose” (1842), „Humoristische Studien” (1844), „Das Blümchen ,Wundervoll”" (1844, Halb Ernst, halb Scherz), ohne Titel (Zum 14ten April 1843. Gedichtet auf Borgorster Hütten. Ueberreicht zum Geburtstage der Frau Pastorin Holst, in Brecklum ${ }^{29}$, ,Seid einig - einig - einig - (Schillers Tell, Act 4, Sc 2)” (Zum Volksfeste der Nordfriesen, den 10ten Juni $1844^{30}$ ), „Begeistrung rief dies Lied ins Leben; Ob sie Wahrheit auch gegeben?" (den 24ten August 1844. Abends), ohne Titel (Zum 3ten September 1844), ,Mein Unglück und mein Trost” (September 1844), „Klage” (2 September 1844).

29 Johann Gottlieb Holst, geb. am 20. März 1802 in Sieseby, war in Breklum seit 1829 Diakon und seit 1837 Pastor. Er starb dort am 16. Januar 1873.

30 Zum Volksfest der Nordfriesen vgl. Johannes Jensen, ,, Nordfriesland in den geistigen und politischen Strömungen des 19. Jahrhunderts (1797-1864)", in: Quellen und Forschungen zur Geschichte Schleswig-Holsteins, Bd 44 (1961), S. $97 \mathrm{ff}$. 
218. Gedichtheft, 8 S., davon S. 3-6 beschrieben, mit folgenden Gedichten: ohne Titel (beginnt mitten in Strophe 4), „Des Leben Traum!”, ohne Titel.

219. Zeitungsausschnitt, Juni 1850, mit einem Gedicht „Der Räuber und sein Kind”, 9 Strophen à 4 Zeilen, unterzeichnet „Neithard”.

2. weiße Mappe: Play presented by the author to the Working-Mens League. 1854

220. „Herr und Sclave. - Schauspiel in 2 Akten am 12ten Februar 1841 (von B. Siegel)", mit einer Eintragung auf der letzten Seite „Dem Arbeiterbunde übermacht als Eigenthum von Fr. Röpenack. Hannover am 26 Julius 1854".

Geschichte eines Sklaven, der von seinem Herrn gedemütigt wird, nachdem er vergeblich an dessen Gerechtigkeitssinn zu appellieren versucht hat. Im 2. Akt nimmt er dafür Frau und Kind seines Herrn in seine Gewalt, ist aber zufrieden, als dieser auf den Knien um Gnade fleht und tötet sich dann selbst.

3. weiße Mappe: Will of John F. Bard naming Wilhelm Weitling executor. 1852 221. Testament John F. Bards vom 16. Dezember 1852, 1 S.

Karton 2 / 4. braune Mappe Memorabilia and biographical sketch

Die 1. weiße Mappe enthält die Erinnerungen Terijon Weitlings. Viele Angaben Wittkes, deren Herkunft bislang unklar war, gehen auf diese Quelle zurück, so z.B. die Ausführungen über Weitlings religiöse Erziehung bis zu dessen zwölften Lebensjahr. ${ }^{31}$ Weiterhin findet sich in dieser Mappe die einzige erhaltene Arbeitsprobe von Weitlings Nähmaschine ( $\mathrm{Nr}$ 225). Die Dokumente der 2. weißen Mappe betreffen größtenteils die Kolonie und den Arbeiterbund. Beachtung verdienen hier die Nebengesetze ( $\mathrm{Nr}$ 243), mit denen die organisatorische Verbindung von Kolonie und Arbeiterbund geregelt werden sollte. Die Liste mit 38 Frauennamen ( $\mathrm{Nr} 244$ ) ist von Interesse, da der Kolonie aus dem Streit zwischen den Geschlechtern große Schwierigkeiten erwachsen sind.

1. weiße Mappe: Memorabilia and biographical sketch

222. Formularvordruck aus Castle-Garden mit handschriftlichen Notizen Weitlings auf der Rückseite (offenbar private Kostenzusammenstellungen).

223. Kleines violettes Büchlein von der New York Journeymen Tailors' protective and benevolent Union, gegründet 1863, revidiert im Februar

${ }^{31} \mathrm{Vgl}$. Wittke, The Utopian Communist, S. 5. 
1864, Mai 1865 und Dezember 1870, New York 1871. Das Büchlein trägt die Aufschrift „Widow of William Weitling”.

Es handelt sich um ein Einzahlungsbuch, in dem Zahlungen, offenbar für die Sterbekasse, von 1871 bis 1. Oktober 1878 quittiert sind. Das Büchlein enthält außerdem die Konstitution und Nebengesetze der Vereinigung.

224. Kleiner blauer Umschlag mit einer Notiz oben links „1114”, darin kleines rosa Büchlein, Titel „Aufnahmebuch des Geschäftsbundes der Arbeiter. Protective Union of the Workingmens-Republic".

225. „Piece of crimped ribbon out of Wilhelm Weitlings machine (by himself)".

226. "On the five questions by Dr. Wittke, in his letter of July 15 th 1946, as to Wilhelm Weitling personalities", 12 S., Handschrift mit Bleistift auf gelbem Papier, oben durch einen Klebestreifen zusammengehalten.

Enthält Antworten auf sieben Fragen.

\section{2. weiße Mappe: Unidentified and miscellany}

227. Transportschein der Western Transportation Company vom 25. Mai 1852; Sendung von Weitling an P. Arnold, St Louis.

228. Einladung zur Subskription der Denk- und Sprachlehre, rückseitig handschriftliche Notizen (offenbar Finanzen des Arbeiterbundes).

229. Rechnung für das Postfach New York 2857, 1. Juli bis 31. Dezember $1854, \$ 2,-$.

230. Reklame- und Bestellzettel für Schmidt-Weißenfels' Bücher, darin besonders hervorgehoben der Titel Zwölf Schneider, der auch Weitling behandelt.

231. Mietquittung und Erklärung der Vermieter von Nr 27 James Street (Druckerei und Redaktion der Republik der Arbeiter).

232. S. 13-15 eines Briefes von Weitling über Streitigkeiten mit Austritt Krisingers und anderer als Konsequenz.

233. 2 Seiten eines Briefes über Druck von Wertpapieren (Tauschbank), Muster für Druck offenbar herausgerissen.

234. Kleiner Notizzettel, New York, 20. November, betr. Fuß- und Beinverletzungen.

235. Schuldschein von Ernst Körbel, ,bezahlt”.

236. Quittung ,1. April 1854 von Weitling \$ 100 zur Ausgabe für Republik der Arbeiter erhalten, Franz Truckswetter".

237. Terminzettel für eine Versammlung am Dienstag 7. Juli betr. Gründung einer Arbeiterzeitung (lateinische Handschrift).

238. Handschriftliche Notiz über einen Artikel aus der Nouvellenzeitung, $\mathrm{Nr}$ 41 von 1854, Feuilleton „Nordwestliche Bilder” von Otto.

239. Brief über Communia (1853), $2 \mathrm{~S}$.

240. Brief über Communia, $2 \mathbf{S}$.

241. Korrespondenz über einen Volkskongreß vom Sonnabend 3. April, 2 S.

242. Antworten auf 14 Fragen mit zahlreichen Randbemerkungen, Dubuque, 18. April 1854, F. Seippel. 
243. Nebengesetze (zur Konstitution der Kolonie Communia).

244. Namensliste, 2 S., enthält 38 Frauennamen.

Karton 2 / 5. braune Mappe

Newspaper Clippings

Diese Mappe enthält 12 Briefumschläge mit Zeitungsausschnitten. Auf den Umschlägen sind, vermutlich in der Handschrift von Terijon Weitling (vgl. Nr 226), Themen notiert, auf die sich die Ausschnitte beziehen. Die Zeitungsausschnitte selbst sind von Wilhelm Weitling gesammelt worden, die Themen der einzelnen Umschläge geben also einen Überblick über dessen Interessengebiete. Die Zeitungsausschnitte zum Thema Astronomy sind der deutsch- und englischsprachigen New Yorker Presse entnommen und, wie alle anderen Ausschnitte, mehrheitlich aus der Zeit zwischen 1857 und 1859. Populärwissenschaftliche Darstellungen der Tagespresse scheinen Weitlings vorrangige Informationsquelle gewesen zu sein, von der wissenschaftlichen Kommunikation war er ausgeschlossen. Auch die im Umschlag Science gesammelten Zeitungsausschnitte beziehen sich mit dem Zodiakallicht auf die Astronomie. Weitlings Interesse für China resultiert möglicherweise daher, daß er im Chamber's Journal vom Oktober 1855 einen Artikel über den chinesischen „Reformator” Wang-gan-schi gelesen hatte, der ihn derart beeindruckte, daß er seinen ersten Sohn nach dem chinesischen Vorbild benannte. Die anderen Umschläge zeigen, daß Weitling auch nach 1855, seinem Rückzug aus der aktiven Politik, ein waches Interesse für die politischen Ereignisse in der Welt hatte. Drei Zeitungsausschnitte liegen lose als Kopie bei, von denen sich zwei auf Weitlings Verhältnis zum Nähmaschinenfabrikanten Singer bzw. auf den Streit unter Singers Erben beziehen. Diese Ausschnitte aus den Jahren 1907 und 1933 hat offenbar Weitlings Familie gesammelt. Der Artikel aus der New Yorker Volkszeitung zeigt, daß Weitling in der amerikanischen Arbeiterbewegung nicht ganz vergessen worden ist, der Verfasser wendet sich vor allem an ,die Socialisten, die Weitling als einen der ihren betrachten".

245. Themen der Zeitungsausschnitts-Sammlungen: Astronomy; Re Working-Mens Colony; Public Lands; Re Universal Language; Science; Re China; Civil War Matters; Re Schiller Monument in London; On Cotton Fields of the World; Re ship sailing; Political; Labor Mass Meeting New York City 1861 and Parade of the Unemployed; „Organization of Workingmen” (loser Zeitungsausschnitt); „Edward Clark Dead at 63 in Cooperstown", aus: Herald Tribune, September 1933 (Kopie); „Zum Kampf um Singer's Millionen”, aus: New Yorker Volkszeitung, 28. März 1907 (Kopie). 
Karton 3

Books, Printed Articles, Diaries

Die Druckschriften des dritten Kartons stehen untereinander in keinem inhaltlichen Zusammenhang. Erläuterungen folgen im Anschluß an das jeweilige Dokument.

246. Tagebuch Weitlings, $43 \mathrm{~S}$., handschriftlich, in den Umschlag eingeritzt „1858 den 6. Mai angelegt von Wm. Weitling”.

Weitlings Tagebuch beginnt im Mai 1858 und in der Absicht, dem ersten Sohn Wang-gan-schi „ein Memorandum aller nur für ihn interessant scheinenden Ereignisse zu hinterlassen". Weitling ist jedoch von Anfang an dazu übergegangen, auch ihn selbst interessierende und betreffende Ereignisse einzutragen. Das Tagebuch ist in unregelmäßigen Abständen geführt, die Eintragungen stammen vom 1. Mai 1858, 21. Oktober 1858, 13. Dezember 1860, 20. August 1862, 3. Februar 1864, 7. Juni 1866, 10. Oktober 1868 und 21.-22. Dezember 1869. Neben pädagogischen und psychologischen Überlegungen zur Kindererziehung finden sich Eintragungen über Religion. Autobiographisches und Familiäres, über Babeuf, Kommentare zu den astronomischen Studien und den Nähmaschinen-Patenten sowie über den Kommunismus. Die Beschreibung der häuslichen Verhältnisse legt ein erschütterndes Zeugnis ab von der bitteren Not der Familie. Auch Karl Marx findet Erwähnung in diesem Tagebuch: Weitling kann den Nutzen der Nationalökonomie als Wissenschaft nicht einsehen, scheint aber die theoretischen Leistungen von Marx, die er auf dessen bürgerliche und intellektuelle Sozialisation zurückführt, anzuerkennen. Für das Verhältnis von Weitling zu Marx könnte auch etwas ganz familiäres Bedeutung haben. Weitling hat seinen fünf Söhnen die Vornamen berühmter Männer gegeben: Wang-gan-schi ist nach einem chinesischen Reformator benannt; Tycho Brahe nach jenem dänischen Astronomen, auf den Weitling in seiner Astronomie rekurriert; Gracchus Babeuf bewunderte schon der junge Weitling; der jüngste Sohn Terijon trägt den Namen von Weitlings Vater. Über den zweitjüngsten Sohn findet sich am Ende des Tagebuchs, nicht in Weitlings Handschrift, die Notiz: „Charles Frederick Weitling was born January 7th 1864 between 5 and 6 . October at 107 Ave. A." Wer sonst wenn nicht Charles Marx und Frederick Engels sollten hier Pate gestanden haben?

247. ,8d and last volume of Jugendbibliothek", Buch in einem PappUmschlag, München 1827, 254 S.

Hierbei handelt es sich möglicherweise um Jugendlektüre Weitlings.

248. J. L. Stiger, ,Stoff zum Nachdenken”, 4 S., gedruckt, mit einem handschriftlichen Kommentar von Weitling.

Diese Schrift läßt sich vorläufig nicht genauer einordnen. J. L. Stiger aus Österreich hatte 1870 Kontakt mit Weitling aufgenommen, da er zur Gründung einer Kolonie aufgerufen hatte und nun Informationen über österreichische Auswanderer in New York wünschte. Nach Weitlings 
Tod korrespondierte er mit dessen Witwe und erbat sich Material für eine Schrift über ihn (vgl. Nr 40-41, 45-48).

249. Gedruckter Text, S. 17-48.

Es handelt sich um den zweiten und dritten Druckbogen der Schrift Das Tausendjährige Reich von Andreas Dietsch. Durch den Verkauf dieser Schrift (wie auch der Garantien) hatte Weitling seine Propagandareise von 1851 finanziert (vgl. Nr 69).

250. „Gedanken über die Gewissensfreiheit. Preisaufgabe der Loge Dovere Diritto in Padua", von Borrutau, 4 S., gedruckt, französisch.

Die Herkunft dieses Textes ist unklar. Borrutau hatte $1868 \mathrm{mit}$ Weitling korrespondiert, weil er eine Gesamtausgabe seiner Schriften plante (vgl. Nr 33). Dabei war es insbesondere um die Frage gegangen, inwieweit Weitlings astronomische Studien in eine solche Gesamtausgabe aufgenommen werden könnten. Im gleichen Brief schlug Borrutau Weitling vor, nach Deutschland zurückzukehren, die Unterstützung der Sozialisten sei ihm gewiß.

251. Gedruckter Text, S. XIX-XXII, Vorwort zur 3. Auflage der Garantien.

252. „Der bewegende Urstoff in seinen kosmo-elektro-magnetischen Wirkungen. - ein Bild des Weltalls den Akademien der Wissenschaften hochachtungsvoll zur Prüfung vorgelegt von Wilhelm Weitling. New York im Februar 1856", Seiten durchgehend numeriert von S. 198 bis S. 214, mit handschriftlichen Korrekturen im Text, Zusatz auf dem Umschlag, in Weitlings Altershandschrift mit Bleistift, „Wm. Weitlings Astronomische Studien, I. Abschnitt", auf der Innenseite des Umschlags mit Bleistift in derselben Handschrift Weitlings ein einseitiger Text.

Über Der bewegende Urstoff schrieb Weitling am 26. Februar 1856 dem Freund Lorenz Petersen: „Ich schickte hier am 8. Februar eines an Humboldt und eines an Chevalier, doch beide nicht - wie sich's gehört - im Briefformat. Ich schickte am 20. Februar mit übersetzten Auszügen ins Französische und Englische eines an l'Institut de France, eines an The Royal Institute of London, eines an den Astronomen Airy in Greenwich, je eines an die Akademie in Berlin, die in Wien, die in München, die in Petersburg und eines an Encke, Direktor der Sternwarte in Berlin. Heute schicke ich eines an die Akademie in Leipzig, eines an die in Breslau und eines an Sir John Herschel. Ich denke, das ist genug, hoffe aber wenig davon." 32 Außerdem hat Weitling ein Exemplar der Schrift an Joseph Henry, den Sekretär der Smithsonian Institution in Washington, gesandt. Die Kaiserliche Akademie der Wissenschaften in Wien hat den Empfang der Schrift am 20. März 1856 bestätigt, weitere Antwortschreiben liegen nicht vor. 1859 hat Weitling zumindest an die Akademien in Wien und Berlin eine weitere astronomische Schrift, die Theorie des Weltsystems, gesandt, die inhaltlich an den Urstoff anschließt. Den Eingang der Theorie des Weltsystems hat Berlin am 8. April 1859 und Wien am 16. Juni 1859 bestätigt, beide Akademien haben jedoch keinerlei Unterstützung gewährt. Barnikol hatte je ein Exemplar

32 Zitiert nach Weitling, Theorie des Weltsystems, a.a.O., S. 5 f. 
des Urstoffs in den Bibliotheken von Berlin, Marburg, Göttingen und Königsberg nachweisen können. Da die Seitenzählung dieser Exemplare mit 197 beginnt, war klar, daß diese Schrift Teil eines umfassenderen Werkes war. Das Auffinden der Denk- und Sprachlehre zeigt, daß der Urstoff die Fortsetzung der Denk-und Sprachlehre ist, die mit der S. 196 und dem Kapitel „Die Urstoffe” endet. Das New Yorker Exemplar des Urstoffs unterscheidet sich von den von Barnikol gefundenen durch einen handschriftlichen Zusatz Weitlings, den dieser für die Nachwelt angefügt hat.

253. „Grundzüge einer allgemeinen Denk- und Sprachlehre. Erstes Buch”, Vorwort S. V-x, Einleitung ohne Seitenangaben, fortlaufender Text S. 14-196.

Die Arbeit an der Denk- und Sprachlehre zieht sich wie ein roter Faden durch Weitlings Leben. Bereits in der Menschheit aus dem Jahre 1838, ausführlicher noch in den Garantien aus dem Jahre 1842, läßt sich der Gedanke einer Universalsprache nachweisen. Während der Haft 1843-44 hat Weitling ausführlicher über eine Klassifikation der Begriffe als „Prüfungsmittel der Wahrheit” nachgedacht. ${ }^{33}$ Im Vorwort zur Denk-und Sprachlehre berichtet Weitling, er sei 1842 durch Gespräche mit Karl Fröbel auf die Notwendigkeit einer einheitlichen Sprache und damit eines überprüfbaren Erkenntnisprozesses gekommen. Wahrscheinlich verwechselt Weitling hier den Pädagogen Karl Fröbel mit dem Buchhändler Julius Fröbel, mit dem er 1843 in Zürich in Kontakt gestanden hat. Nach der Haftentlassung hat Weitling seit dem Sommer 1844 ,ein Jahr lang unausgesetzt" an der Denk- und Sprachlehre gearbeitet. ${ }^{34}$ Auch 1846 hat er an seiner ,Systemarbeit”, der Denk- und Sprachlehre, gearbeitet und vergeblich versucht, diese zu publizieren ${ }^{35}$ Die nächste Arbeitsetappe ist für den Aufenthalt in Hamburg 1848-49 nachgewiesen, nachdem Weitling aus Berlin ausgewiesen worden war. Als Weitling im Frühjahr 1849 auch aus Hamburg verwiesen werden sollte, setzten sämtliche fortschrittlichen Vereine, Bürger-, Arbeiter- und Bildungs-Vereine Hamburgs eine Protestresolution auf, in der betont wird, daß Weitling als ein Mann der Wissenschaft an einer ,Sprachlehre" arbeitet ${ }^{36}$ In Hamburg erschien auch ein Prospekt zur Werbung von Subskribenten für die Denk- und Sprachlehre, die in Hamburg für Alexander von Humboldt angefertigte Klassifikation des Universums ist ein Extrakt der Denk-und Sprachlehre. In der Republik der Arbeiter ist die Denk - und Sprachlehre dreimal angekündigt worden, in der $\mathrm{Nr} 9$ vom 14. Juni 1851, in der Nr 29 vom 15. Juli 1854 und schließlich in einer der letzten Nummern, der vom 17. März 1855. Im Hamburger Prospekt von 1848/49 war von 25 Druckbogen die Rede, 1855 sind 12-13 Bogen

${ }^{33}$ Weitling, Gerechtigkeit, a.a.O., S. 145.

$34 \mathrm{Nr} 253$, S. vill.

35 Vgl. den Brief Weitlings an Moses Heß vom 31. März 1846, in Barnikol, Weitling der Gefangene und seine „Gerechtigkeit”, a.a.O., S. 270.

36 Vgl. ebd., S. $254 f$. 
angekündigt, von denen 8 Bogen ,im großen Octavformat” bereits gedruckt sein sollen. Während Schlüter berichtet hatte, die zum Teil gedruckte Denk-und Sprachlehre sei von Weitling 1869 verbrannt worden, hat Wittke eine ausführliche Zusammenfassung des Inhalts gegeben. ${ }^{37}$ Die Grundzüge einer allgemeinen Denk-und Sprachlehre liegen in 196 Seiten komplett in einem einzigen Exemplar vor, in dem Weitling handschriftlich einige wenige Korrekturen hinzugefügt hat. In dieser Schrift ist die Klassifikation des Universums verarbeitet, eine neue Universalsprache entwickelt und die Brücke zur eingehenderen Beschäftigung mit der Astronomie geschlagen; darüberhinaus beschäftigt sich Weitling mit Wahrnehmungsphysiologie und erkenntnistheoretischen Fragen, so daß die Denk-und Sprachlehre als sein philosophisches Hauptwerk betrachtet werden kann. Im Vorwort weist er noch einmal auf den sozial und politisch motivierten Ausgangspunkt bei der Beschäftigung mit der Sprache und mit dem Denken hin: Weitling will verhindern, daß Nebelphilosophen, Demagogen und Sophisten, die der Sache des Proletariats bestenfalls mit dem Kopf, nie jedoch mit dem Herzen zugetan sind, sich an die Spitze der Arbeiterbewegung stellen und das Proletariat von einer Geistesaristokratie angeführt wird.

254. Weitling, ,Account Book” 1851-53, Einband von der New York Public Library, einige Seiten mit Zeitungsausschnitten von 1876 überklebt, einige Seiten herausgerissen, 82 Seiten des Originals erhalten, auf S. 72 und 142-49 astronomische Studien, handschriftlich.

Im Account Book hat Weitling sehr genau Buch geführt über die Mitgliedschaft des Arbeiterbundes. Es kann davon ausgegangen werden, daß für den fraglichen Zeitraum (1851-53) alle Mitglieder erfaßt waren, wobei eine Unterteilung nach Städten vorgenommen wurde. Es entstehen allerdings Lücken durch fehlende und überklebte Seiten.

255. „Tagebuch der Central Geschaefte des Arbeiterbundes oder Verbrüderung Workingmens League angelegt und fortgeführt seit dem 1. März von W. Weitling", darin sonstige Notizen auf S. 95-101, handschriftlich.

Im Unterschied zum Account Book geht es hierin um die Einnahmen und Ausgaben des Arbeiterbundes, über die Weitling sehr gewissenhaft Buch geführt hat. Indirekt dürften sich hier einige Aufschlüsse über die Tätigkeit des Bundes ergeben. Beide Tagebücher hat Weitling außerdem für private Notizen benutzt, u.a. für seine astronomischen Studien.

37 Wittke, The Utopian Communist, S. 294-97. 